\title{
Ileal gastrointestinal stromal tumors mimicking gynecological masses: a single institutional experience
}

Giulia Dondi MD1, Anna Myriam Perrone MD PhD1, Margherita Nannini MD2, Marco Tesei MD1, Maristella Saponara MD2, Antonio De Leo MD3, Donatella Santini MD3, Maria Pantaleo MD PhD2, Pierandrea De Iaco MD1

1 Gynecological Oncology Unit, Sant'Orsola-Malpighi Hospital, University of Bologna, Italy

2 Specialized, Experimental and Diagnostic Medicine, Sant'Orsola-Malpighi Hospital, University of Bologna, Italy

3 Pathology Service, Addarii Institute of Oncology, Sant'Orsola-Malpighi Hospital, University of Bologna, Italy

Introduction

Gastrointestinal stromal tumor (GIST) is the most common mesenchimal neoplasm of the gastrointestinal tract. The small intestine is the second predominant site of origin of GIST (1). This neoplasia has a wide spectrum of clinical presentations and rarely can be diagnosed as an asymptomatic pelvic mass accidentally detected by transvaginal ultrasound and thus wrongly diagnosed as a gynaecologic tumor in female patients. To date, in literature 35 cases of ileal GIST mimicking gynecologic masses have been described (2-10)

The aim of this study is to describe the clinical and trans-vaginal sonographic appearance of an ileal GIST mimicking a gynecologic tumors in five patients referred to our Institution, in order to better recognize this rare clinical presentation.

Patients and methods

We retrospectively reviewed 64 female patients with ileal GIST, collected since 2001 to 2017 in our GIST database. Among those 64 female patients with ileal GIST, we selected 5 patients presented with a pelvic mass and referred to a gynecological consultation at first. Characteristics of patients are listed in Table $\mathbf{1 .}$

Results

Clinically, one patient was asymptomatic and the mass was occasionally found with a routine transvaginal ultrasound, two presented with abdominal pain, one with melena and one with a left inguinal swelling. In all cases CA 125 was negative or mildly increased $(<170 \mathrm{U} / \mathrm{ml})$.

At trans-vaginal ultrasound exam all the lesions were predominant solid, dishomogeneous and hypoechoic (Figure 1A and 1B).

The mean diameter was $68 \mathbf{~ m m}$. In four cases the lesions were described as "Iobulated masses". Cystic areas due to necrosis were described in one patient. In all cases no acoustic shadows were observed. In three cases the mobility towards other pelvic structures was reduced and in four cases the masses were localized in the right pelvis mimicking right ovarian lesions. All cases showed a high vascularization at Power Doppler (Fig $2 \mathrm{~A}$ and 2 B). A laparotomic ileal resection was performed in all cases. Hysterectomy and bilateral salpingooophorectomy were performed in 2 cases and left salpingo-oophorectomy in one. The histologic exam revealed an ileal GIST with a mitotic index ranging from $<5 / 50$ HPF to $19 / 50 \mathrm{HPF}$ (median: 5/50 HPF) with bilateral ovarian metastases in one patient with an advanced disease (11).

Conclusion

In our experience the clinical presentation of ileal GIST was heterogeneous and nonspecific and does not permit a differential diagnosis between GIST and ovarian tumor. GIST shows transvaginal sonographic peculiar features. The mass appears solid, irregular, hypo-echoic with small anechoic parts, without acoustic shadows, highly vascularized and frequently dislocated in the right pelvis. Normal or mild serum levels of CA $125(<170 \mathrm{U} / \mathrm{ml})$ and absence of free fluid in the pouch of Douglas can be considered ancillary features for suspicion of GIST. Lastly a gyneacologic evaluation should be proposed in patients with a history of GIST because of the possibility of metastases to the genital tract (11).

References

1.Biasco G, Velo D, Angriman I, Astorino M, Baldan A, Baseggio M, et al. Gastrointestinal stromal tumors: report of an audit and review of the literature. Eur J Cancer Prev. 2009 Apr;18(2):106-16.

2.Dayan E, Zhu H, Copperman AB, Lewis S, Kamath A. Gastrointestinal stroma tumor presenting as a right adnexal mass with histopathologic correlation. Clin Imaging 2017;44:97-100

3.Ijeri SK, Rathod PS, Kundargi R, Pallavi VR, Shobha K, Shankaranand, Vijay CR, Uma Devi K, Bafna UD. Gastrointestinal Stromal Tumor Mimicking as Ovarian Tumor in Gynaecologic Oncology. Indian J Surg Oncol 2016;7:56-61. 4.Karaca N, Akpak YK, Tatar Z, Batmaz G, Erken A. Gastrointestinal Stromal Tumor: May Mimic Adnexal Mass. Glob J Health Sci 2015;8:20-6

5.Muñoz M, Ramirez PT, Echeverri C, Alvarez LG, Palomino MA, Pareja LR. Gastrointestinal stromal tumors as an incidental finding in patients with presumptive diagnosis of ovarian cancer. J Gynecol Oncol 2012;23:48-52. 6.Teoh WC, Teo SY, Ong CL. Gastrointestinal stromal tumors presenting as gynecological masses: usefulness of multidetector computed tomography. Ultrasound Obstet Gynecol. 2011;37:107-9.

7.Angioli R, Battista C, Muzii L, Terracina GM, Cafà EV, Sereni MI, Montera R, Plotti F, Rabitti C, Panici PB. A gastrointestinal stromal tumor presenting as a pelvic mass: A case report. Oncol Rep 2009;21:899-902

8.Pinto V, Ingravallo $G$, Cicinelli $E$, Pintucci $A$, Sambati GS, Marinaccio $M$, D'Addario V. Gastrointestinal stromal tumors mimicking gynecological masses on ultrasound: a report of two cases. Ultrasound Obstet Gynecol 2007;30:35961.

9.Morimura $\mathrm{Y}$, Yamashita N, Koyama N, Ohzeki T, Takayama T, Fujimori K, Sato A. Gastrointestinal stromal tumor mimicking gynecological disease. Fukushima J Med Sci 2006;52:21-8.

10.Zighelboim I, Henao G, Kunda A, Gutierrez C, Edwards C. Gastrointestina stromal tumor presenting as a pelvic mass. Gynecol Oncol 2003;91:630-5

11.De Leo A., Nannini M., Dondi G., Santini D., Urbini M., Gruppioni E., De Iaco P., Perrone A.M., Pantaleo M.A. Unusual bilateral ovarian metastases from ileal gastrointestinal stromal tumor (GIST): a case report. BMC Cancer. 2018 Mar 16;18(1):301

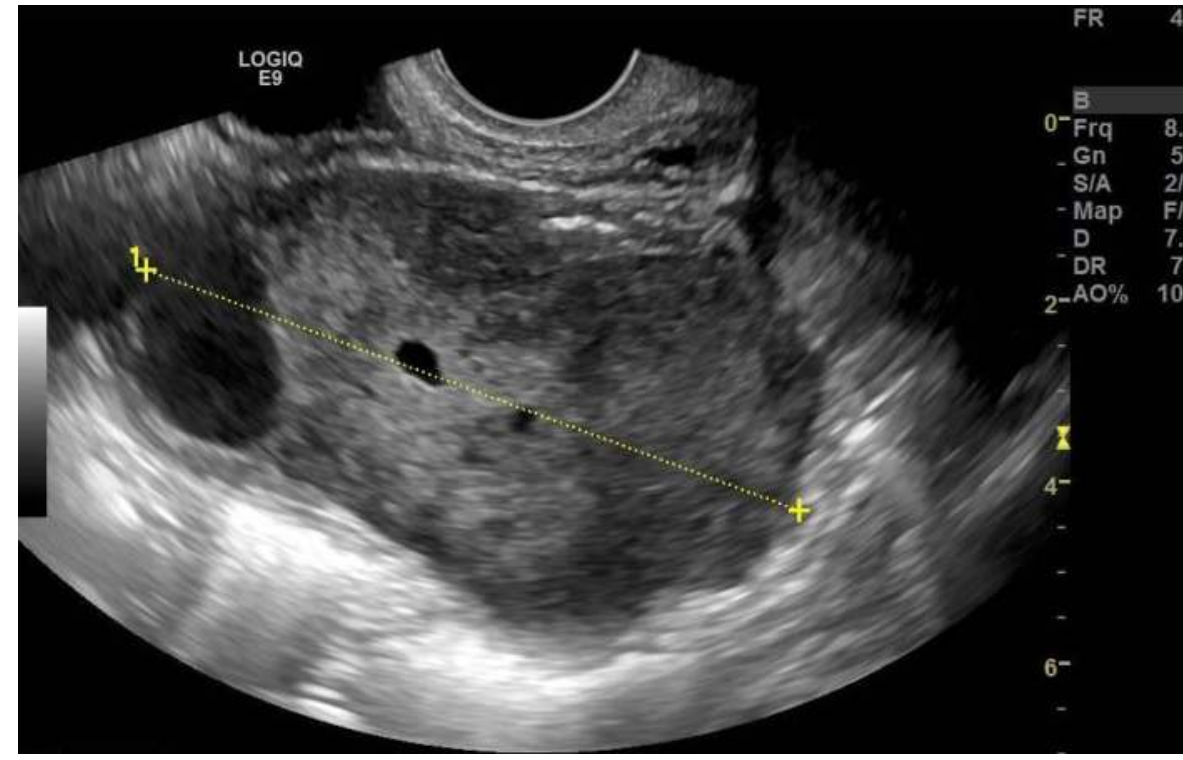

Fig.1 A: Trans vaginal ultrasound image of the right pelvic mass (Case 3)

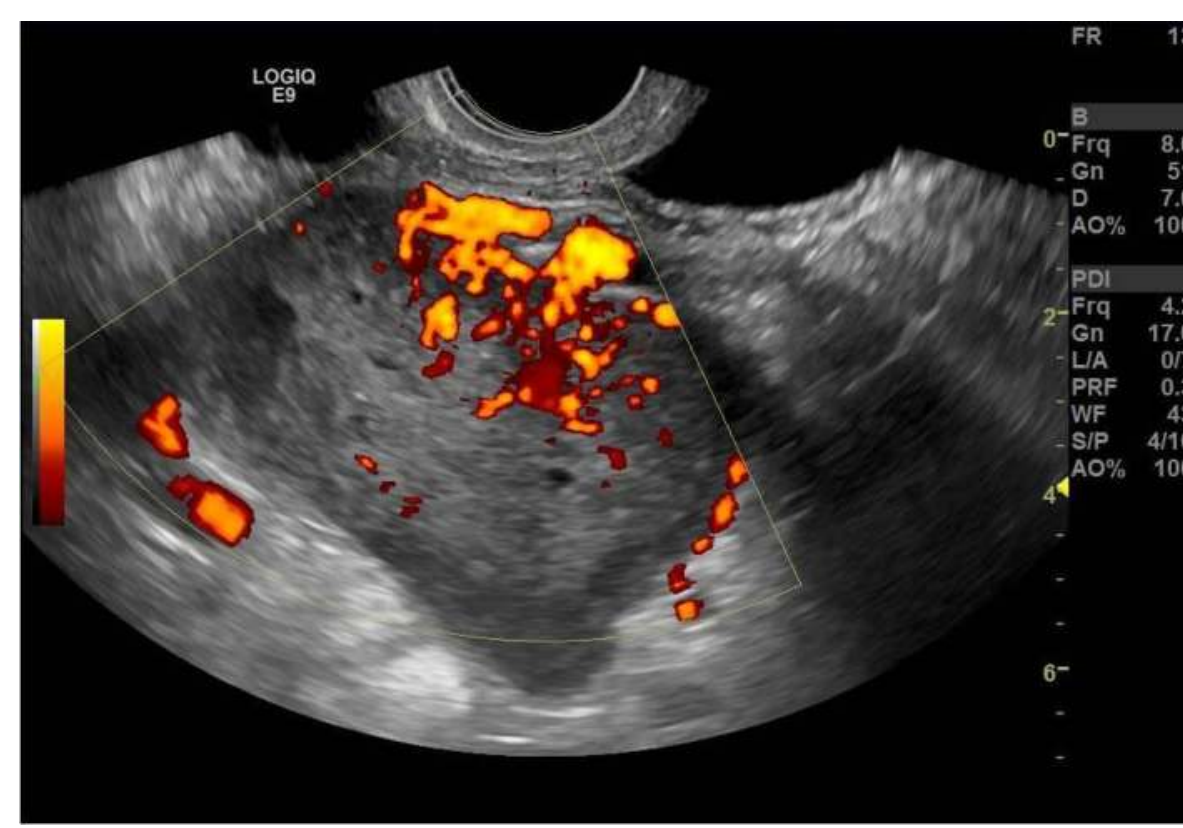

Fig. 1 B: Study of the vascularization of the pelvic mass using transvaginal Doppler ultrasonography (Case 3)
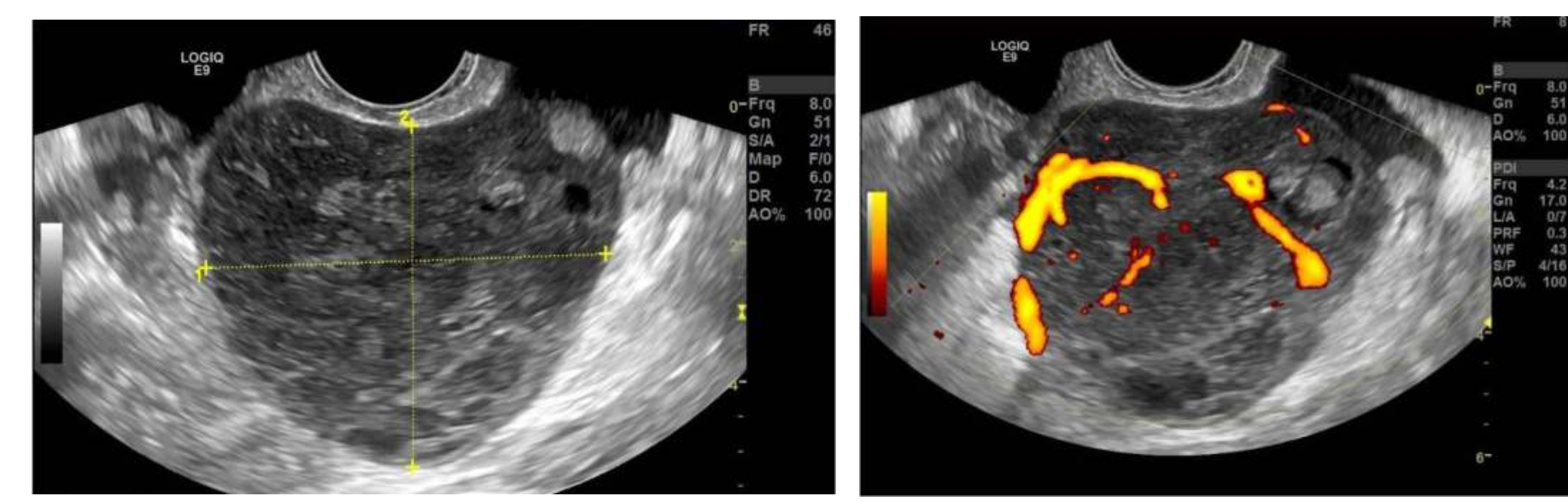

Fig.2 A: Trans vaginal ultrasound image of the right pelvic mass (Case 4).

Fig. 2 B: Study of the vascularization of the pelvic mass using transvaginal Doppler ultrasonography (Case 4)

Table 1: Patients' characteristics

\begin{tabular}{|c|c|c|c|c|c|c|c|c|}
\hline Patient & Age & $\begin{array}{c}\text { Clinical } \\
\text { presentation }\end{array}$ & $\begin{array}{l}\text { Surgical } \\
\text { Management }\end{array}$ & $\begin{array}{l}\text { Disease } \\
\text { status }\end{array}$ & $\begin{array}{l}\text { Size } \\
(\mathrm{cm})\end{array}$ & $\begin{array}{l}\text { Mitotic } \\
\text { index }\end{array}$ & $\begin{array}{l}\text { Molecular } \\
\text { analysis }\end{array}$ & $\begin{array}{l}\text { Follow-up } \\
\text { status }\end{array}$ \\
\hline Case 1 & 31 & $\begin{array}{l}\text { Melena, } \\
\text { anemia }\end{array}$ & Ileal resection & Localized & 7,5 & $5 / 50 \mathrm{HPF}$ & $\begin{array}{l}\text { exon } 11 \text { KIT [c.1657_1668del } \\
\text { (pY553_Q556)] }\end{array}$ & $\begin{array}{l}\text { Alive without } \\
\text { disease } \\
\text { (OS: } 49 \text { months) }\end{array}$ \\
\hline Case 2 & 77 & $\begin{array}{l}\text { Left inguinal } \\
\text { swelling }\end{array}$ & $\begin{array}{l}\text { lleal resection } \\
\text { hysterectomy } \\
\text { bilateral salpingo } \\
\text { oophorectromy } \\
\text { asportation of } \\
\text { peritoneal lumps }\end{array}$ & Advanced & 17 & 9/50HPF & $\begin{array}{l}\text { exon } 9 \mathrm{KIT} \\
\text { (c.1509_1510insGCCTAT) }\end{array}$ & Dead \\
\hline Case 3 & 53 & $\begin{array}{l}\text { Abdominal } \\
\text { pain }\end{array}$ & Ileal resection & Localized & 8 & 5/50HPF & KIT/PDGFRA WT & $\begin{array}{l}\text { Alive without } \\
\text { disease } \\
\text { (OS: } 16 \text { months) }\end{array}$ \\
\hline Case 4 & 76 & $\begin{array}{l}\text { Abdominal } \\
\text { pain }\end{array}$ & $\begin{array}{l}\text { Ileal resection, left } \\
\text { salpingo-oophorectomy } \\
\text { omentectomy }\end{array}$ & Localized & 6,5 & $<5 / 50 \mathrm{HPF}$ & $\begin{array}{l}\text { exon } 11 \mathrm{KIT} \\
\text { [p. V559D (c.1676T>A)] }\end{array}$ & $\begin{array}{l}\text { Alive without } \\
\text { disease } \\
\text { (OS: } 12 \text { months) }\end{array}$ \\
\hline Case 5 & 50 & None & $\begin{array}{l}\text { leal resection } \\
\text { hystectomy } \\
\text { bilateral salingo } \\
\text { oophorectomy } \\
\text { douglas pouch and } \\
\text { pelvic peritonectomy }\end{array}$ & Advanced & 7 & 19/50HPF & $\begin{array}{l}\text { exon } 11 \text { KIT [p.M552_W557 del } \\
\text { (c-1653_1670del)] }\end{array}$ & $\begin{array}{l}\text { Alive without } \\
\text { disease } \\
\text { (OS: } 7 \text { months) }\end{array}$ \\
\hline
\end{tabular}

